\title{
Occupational factors and unexplained physical symptoms
}

\author{
Matthew Hotopf
}

Liaison psychiatrists are frequently asked to see people with chronic, debilitating and unexplained physical symptoms, in particular chronic pain and chronic fatigue. People with these conditions have often seen many doctors and received multiple medical investigations, but rarely have had any effective treatment or even explanation for their symptoms. There are many reasons for focusing on occupational factors in this patient group. First, many people may attribute their symptoms to workplace exposures. For example, back pain may commonly be put down to accidents; upper limb disorders to use of keyboards or repetitive manual tasks; and headaches and fatigue to visual display units or air conditioning. These attributions have prognostic and legal implications, and are likely to affect the course of treatment. Second, for those who have had prolonged sickness absence, it is often necessary to make decisions regarding return to work or to help them negotiate the often lengthy and complex medical retirement process. This may be a major stumbling block in managing such people. Finally, psychiatrists are frequently invited to write medico-legal reports regarding the role of occupational factors in causing unexplained physical symptoms. This article aims to address these issues and provide some practical guidance to clinicians.

\section{Definitions}

Most medical specialists are familiar with patients who complain of physical symptoms, but in whom no defined physical cause can be found. A number of systems are used to describe such people (Box 1). Physicians tend to classify medically unexplained symptoms according to the organ systems affected, and each medical speciality has its own syndrome. Rheumatologists now recognise the diagnosis of fibromyalgia, which consists of chronic unexplained pain, tenderness and fatigue. Gastroenterologists recognise irritable bowel syndrome and some cardiologists refer to 'atypical chest pain'. These syndromes are useful for physicians and patients in that they are based simply on a list of physical symptoms, and make no assumptions regarding aetiology. However, there is considerable overlap between each syndrome.

Psychiatrists have tended to use systems which ignore the organ system affected but emphasise psychopathology. These are described in ICD-10 (World Health Organization, 1992) and DSM-IV (American Psychiatric Association, 1994) as the somatoform disorders. These disorders include somatisation disorder, hypochondriasis and

Box 1. Classifying medically unexplained symptoms

As categories

ICD-10 or DSM-IV somatoform disorders (e.g. somatisation disorder)

Medically unexplained syndromes (e.g. irritable bowel syndrome)

As a process

Somatisation

"... a tendency to experience and communicate somatic distress and symptoms unaccounted for by pathological findings, to attribute them to physical illness and to seek medical help for them." (Lipowski, 1988)

Matthew Hotopf is a Medical Research Council Training Fellow (Department of Psychological Medicine, Institute of Psychiatry, 103 Denmark Hill, London SE5 8AZ) whose research interests include medically unexplained symptoms, chronic fatigue syndrome, depression and occupational health. 
chronic pain syndromes. Again, the specificity of most of these disorders is low, and they have been criticised for only reflecting the most severe 'tip of the iceberg'.

The approach traditionally adopted in primary care settings is to see unexplained symptoms in the context of the doctor-patient relationship. This process has sometimes been referred to as somatisation (Goldberg \& Bridges, 1988; Lipowski, 1988), a term which has theoretical limitations as it implies the conversion of psychological distress into physical symptoms. Perhaps the most useful approach is simply to describe unexplained symptoms for what they are, without attempting to invoke unproven processes or false categories (Mayou et al, 1995).

Whichever method of defining symptoms is used, they are common. Population surveys find that for healthy adults having at least one physical symptom is the rule not the exception. Symptoms like fatigue, headache and back pain all individually have prevalences of between 25 and $50 \%$, depending on how they are defined (Hannay, 1978; Crooket al, 1984; Kroenke et al, 1994). Studies based in medical settings find that the majority of physical symptoms are not attributable to defined organic causes (Kroenke \& Mangelsdorff, 1989).

Chronic unexplained symptoms are extremely disabling and place an enormous burden on industry. The most common single cause of certified sickness absence is back pain, which accounts for over $10 \%$ of sick days (Frank, 1993).

\section{Non-workplace risk factors}

\section{Psychiatric disorder}

By far the most studied and best understood risk factor for unexplained symptoms is psychiatric disorder (see Box 2). For example, over half of the people with chronic fatigue syndrome or fibromyalgia may have a recognisable psychiatric disorder, the most common of which is depression (Wessely \& Powell, 1989). This relationship does not seem to be due solely to the consequences of physical symptoms, since control groups of physically ill subjects have generally lower levels of psychiatric morbidity.

The relationship between single physical symptoms and psychiatric disorder is also strong. A number of community studies have shown powerful crosssectional relationships between physical symptoms and psychiatric disorder. Our work on six common physical symptoms (back ache, rheumatism, chest pain, abdominal pain, dizziness and headache) showed these were up to six times more common in those with psychiatric disorder. As a general rule, the more physical symptoms an individual complains of, the greater their likelihood of psychiatric disorder.

Of course, the cross-sectional relationship between symptoms and psychiatric disorder, does not tell us which came first. However, a number of studies of individual symptoms and groups of symptoms suggest that psychiatric disorder is a predictor of developing new physical symptoms (Croft et al, 1996). Recent work using the Medical Research Council National Survey of Health and Development indicates that the relationship operates in a reciprocal manner: several physical symptoms predict the onset of new psychiatric disorder, but psychiatric disorder is also a predictor of new physical symptoms (further details available from the author upon request).

Many people with chronic unexplained physical ailments put them down to a well-defined physical illness or trauma caused by accidents. In individual cases there often seems to be a clear temporal link, between such acute episodes and chronic symptoms. One consistent finding is that irrespective of the causal role of the acute illness, the people most at risk of going on to develop chronic physical symptoms are those who have a past history of depression. This applies to patients who develop post-herpetic neuralgia (Dworkinet al, 1992), those who develop chronic fatigue following viral infections (Hotopf et al, 1996) and those who develop post-enteritis irritable bowel symptoms (Gwee et al, 1996). Psychiatric disorder is therefore a predisposing and possibly a precipitating factor in the development of unexplained symptoms. There is also considerable evidence to suggest it has a role in perpetuating symptoms. For example, people with chronic fatigue who are depressed or have a past history of depression, do less well than those who have no psychiatric disorder (Joyce et al, 1997).

Psychiatric disorder is thus an important risk factor for unexplained symptoms, but it is not a full explanation. Most sufferers from unexplained symptoms have no history of psychiatric disorder, and in population terms even mild psychiatric disorder can account for at most $40 \%$ of all symptoms (further details available from the author upon request). Thus, there is a need to explore other psychosocial explanations.

\section{Early experience}

Early childhood experience has long been a popular explanation for unexplained physical symptoms. In order to make the diagnosis of 
Box 2. Association between psychiatric disorder and physical symptoms

There is a strong cross-sectional relationship between individual symptoms and common mental disorders.

The more physical symptoms reported, the greater the likelihood of psychiatric disorder.

Defined physical illnesses are strongly associated with depression and anxiety.

Psychiatric disorder is a predictor for:

- later development of unexplained symptoms

- development of chronic symptoms after an acute physical illness

- remaining unwell in chronic pain or chronic fatigue.

somatisation disorder (the most severe of the somatoform disorders) the history of physical symptoms must be present from early adulthood. Therefore, it is not surprising that researchers have looked for childhood experiences as a possible explanation. Broadly, two groups of risk factors have been described. Some, like childhood abuse and neglect or other aspects of unsatisfactory parenting, are probably risk factors for most psychiatric disorders. However, the relationship between chronic pelvic pain and childhood sexual abuse does seem inherently understandable, and reflects the notion from classical psychodynamic theory that 'hysterical' symptoms often have an underlying psychic meaning.

The other group of early risk factors are probably more specific to the development of unexplained symptoms. These risk factors relate to the experience of illness in childhood: either in the individual or in their close family. The South London Somatisation Study (Craig et al, 1993) illustrated this. Primary care attenders were divided into 'somatisers' and 'psychologisers' and then compared on a range of childhood experiences. A similar proportion in both groups reported unsatisfactory experiences of parenting, and these were higher than controls with physical disease. This suggests that this risk factor is nonspecific, and predicts psychiatric disorders across the board. What distinguished the somatisers from psychologisers was their tendency to report either having been sickly as a child or having close relatives who were ill throughout their childhood. Using the MRC National Survey of Health and Development, we have been able to demonstrate this effect using prospective data (further details available from the author upon request). Adults who report multiple physical symptoms have parents who reported more than twice as many physical symptoms when the subjects were in their teens. Childhood physical illness itself was not related to later physical symptoms, which should be reassuring to paediatricians.

\section{Cognitive and behavioural theories}

The above studies suggest that previous experiences of illness and the sick role may play a part in an individual's predisposition to interpret physical symptoms in a certain light if they have an acute illness. It may be that such interpretation leads to a pattern of behaviour which perpetuates symptoms, by leading to fear and avoidance. Thus, adults who come from a sickly family may become more anxious when they experience symptoms which others might ignore, and this may lead to a series of secondary behaviours.

This is illustrated by the case of chronic fatigue syndrome. A consistent finding of several outcome studies is that people who state that their illness is caused by physical factors (typically a viral illness) are less likely to improve (Joyce et al, 1997). These types of external attributions for symptoms which have much in common with depression appear to protect the individual from guilt or worthlessness (Powell et al, 1990), but also may predict a number of maladaptive behaviours. Classically people suffering from chronic fatigue syndrome undergo a cycle by which days of reduced activity are followed by frustration and an attempt to increase activities to premorbid levels. This leads to the re-experiencing of physical symptoms (probably because of physical de-conditioning) and may be interpreted by the sufferer as a sign of relapse, caused by their body still being attacked by viral infection. It is easy to see how this sort of pattern can lead to a further reduction in activities and further avoidance. It is also likely that similar mechanisms operate in chronic pain syndromes. For example, if a sufferer from back pain were to interpret his or her symptom as evidence that their spine was crumbling (a belief which might be reinforced if their grandmother had osteoporosis), they are more likely to respond to the pain by avoidance and rest.

\section{Iatrogenesis}

No discussion of medically unexplained symptoms is complete without mention of the medical profession's role in perpetuating old symptoms and causing new ones. Sadly, there are very few 
studies that have systematically examined the effects of medical management on people with medically unexplained symptoms. What is clear is that a large number of patients experience multiple physical investigations, referrals and treatments which, with the benefit of hindsight, may cause more problems than they solve (Kouyanou et al, 1997). A common difficulty is that additional investigations in these people may lead to mild and probably unrelated pathology being discovered. Many people suffering from lower-back pain have a lumbar spine X-ray which demonstrates 'mild degenerative changes'. While a clinician may be inclined to play down such changes, many people will interpret them as evidence of severe permanent damage, and this will be a further contributor to fear and avoidance.

\section{Cultural and societal factors}

Attributions and attitudes to physical symptoms develop in a social context, and it is probable that the workplace plays an important role in determining how an individual appraises a common symptom. The most extreme example of social contagion is mass hysteria, in which a collection of physical symptoms may spread across a workplace (or, more commonly, a school). Many of the symptoms in mass hysteria are those of acute anxiety, but there is frequently an added dimension, namely the attribution made by sufferers and outsiders. It is common for episodes of mass hysteria to be attributed to a gas leak, infection or some other unseen, unpredictable and alarming cause. It is not difficult to see how such an attribution can lead to escalating anxiety, symptom monitoring and illness. Most of those affected in mass psychogenic outbreaks are normal individuals overtaken in a mass panic. The best response is firm reassurance by the authorities, and probably closing the workplace for a brief period. These sorts of cultural attributions may also be responsible for some of the occupational syndromes which have been described.

\section{Workplace-specific rick factors}

\section{Physical aspects of the workplace}

A number of workplace activities may lead directly to physical symptoms. Thus, in general, blue collar workers experience more aches and pains than white collar workers, and these are likely to be due to the nature of their work. Those who work standing or lifting are at increased risk of lower-back pain (Svensson \& Andersson, 1989; MacFarlane et al, 1997). Although there may be no pathological damage, the association between this sort of activity and the pain is indisputable, and it is not necessary to invoke complex pathophysiological mechanisms. Other examples are the associations between neck and shoulder pain with activities involving holding the arms above the head for long periods. Whether these activities in themselves lead to chronic or permanent damage is discussed below.

More contentious and difficult to study is the causative role of accidents in unexplained physical symptoms. It is certainly true that workers are likely to blame accidents in the workplace, though perhaps less than might be expected. Under $10 \%$ of persistent pain sufferers mention a workplace accident as the cause (Crook et al, 1984). None the less, accidents are a common reason for personal injury claims against employers.

\section{Psychosocial aspects of the workplace}

If psychiatric disorder is associated with unexplained physical symptoms, it is not surprising that psychosocial adversity in the workplace may also be important. A systematic review has shown that monotonous work, with high perceived workloads and time pressure, is related to musculoskeletal disorders, and this has been demonstrated using both cross-sectional and prospective data (Bongers et al, 1993). A good example is the Boeing study (Bigos et al, 1991). A cohort of aircraft manufacturers were followed and new episodes of back pain monitored. The workers at greatest risk were those who at the start of the study reported low work satisfaction, and scored highest on the Minnesota Multiphasic Personality Inventory's (Hathaway \& McKinley, 1967) 'hysteria' scale (which, in effect, is an inventory of physical symptoms). Job satisfaction is not far from the fashionable (but under-studied) notion of stress in the workplace (Hotopf \& Wessely, 1997). Workers with low job satisfaction are presumably more likely to report symptoms of stress, and it is certainly common for people with medically unexplained symptoms to report having felt under increased strain at work, or suffered bullying in the months leading up to their symptoms.

\section{Work-related disorders}

A number of disorders characterised by unexplained physical symptoms which have been attributed to workplace exposures have been described. These 
include 'sick building syndrome', 'repetitive strain injury', 'techno stress' and 'environmental illness'. These disorders are characterised by unexplained physical symptoms which are common in all settings: headaches, fatigue, rashes, diffuse pain. The symptoms are attributed specifically to a work-related physical cause. Legal action is often taken against the employer, and media attention may be intense.

The symptoms the sufferers complain of are genuine and disabling, but the relationship between the symptom and alleged cause is usually weak. The linking of alleged cause and symptom in the name of such disorders is dangerous for several reasons. If the syndrome is defined according to the presumed cause, this stifles research into causal mechanisms that are not related to work, which may be just as important. Also, people with these diagnoses may be very resistant to alternative explanations. We have seen in chronic fatigue syndrome how certain attributions may lead to worse outcome, and it is likely that these sorts of attribution, which are external and physical, may anticipate a cycle of fear and avoidance. Finally, using names which imply a direct association between the workplace exposure and the physical symptom may encourage misguided and lengthy legal action.

There are a number of workplace and nonoccupational associations of unexplained physical symptoms. Early experiences may predispose the individual to interpret symptoms in a certain light. Past psychiatric disorder may make the individual vulnerable to react to acute illnesses or accidents in a maladaptive manner. Certain job pressures and dissatisfaction may also predict a maladaptive response to symptoms. Physical characteristics of work, or workplace accidents, may cause acute symptoms which will be responded to according to predisposing factors.

\section{Management}

The management of medically unexplained symptoms has been described in full elsewhere (Mayou et al, 1995). The main aspects of management are described in Box 3.

A number of specific psychotherapeutic techniques have been used for unexplained physical symptoms. Recent randomised controlled trials suggests cognitive-behavioural therapy is helpful in chronic fatigue syndrome (Sharpeet al, 1996; Dealeet al, 1997) and unexplained symptoms, and that psychodynamic therapy may be helpful in irritable bowel syndrome (Guthrie et al, 1993).
Box 3. Aspects of management

Engagement of the patient: overcoming their suspicions of seeing a psychiatrist

Take physical symptoms seriously, and use them as a means of engaging with the patient Take a collaborative approach

Link psychological distress with symptoms

Diagnose and treat co-existent psychiatric disorder

Involve partners and family

Negotiate a graded increase in activities

Explore fears surrounding physical symptoms

\section{Specific problems in management}

Patients who have been unwell for several months may find it difficult to return to full activities immediately, even if they are symptomatically improved. Whereas there is often a strong clinical indication to recommend a graded return to work, it is more usual to be faced with a person who is on the verge of being dismissed on grounds of ill-health, and may have become involved in acrimonious quarrels with their personnel department. They may also be conscious of ill-feeling among fellow workers because of their prolonged sickness absence. Despite these obstacles, it may be possible to instigate workplace rehabilitation (Mountstephen \& Sharpe, 1997). This involves liaison between the treating psychiatrist/psychologist and the occupational health physician, who is in the key position to ensure that rehabilitation can take place. These types of schemes have not been formally evaluated.

\section{Medical retirement}

One of the most serious decisions to be made may relate to medical retirement (Mountstephen \& Sharpe, 1997). This is often dealt with by occupational physicians and general practitioners, but the psychiatrist may have an important role. Clinical experience and some research suggests that the decision regarding medical retirement is often made on an arbitrary basis. In some cases employees have been retired before any proper attempt has been made at treatment. In other cases employers operate rigid policies which would preclude sufferers of chronic pain syndromes or chronic fatigue syndrome from ever receiving medical retirement, as these conditions are not perceived as permanently disabling.

Ideally, people should have the opportunity to be fully treated before any decision regarding medical retirement has been made. However, it is common to see people who are in the process of 
negotiating medical retirement and if, as is often the case, the person seems unlikely to be able to return to their previous job, it is probably better to assist in medical retirement prior to treatment. In a trial of cognitive-behavioural therapy for chronic fatigue syndrome, being involved in negotiating medical retirement was the worst prognostic indicator (Deale et al, 1997). It is often simply impossible to get further in treatment until the issue has been resolved. Therefore, the best approach is a pragmatic one, involving detailed discussion with the patient, their general practioner and, if possible, the occupational health department.

\section{Insurance}

Another common problem relates to insurance companies' reimbursement for sickness absence (Aylward \& Locascio, 1995). While the insurance companies are aware of high levels of fraud (for example, individuals who claim insurance for illhealth, but are doing another job) and understandably want to protect themselves against this, their approach to people with unexplained physical symptoms is often unsympathetic. For example, insurers often rely on abnormal physical investigations to validate the suffering reported by claimants. Psychiatrists are sometimes asked to prepare reports for the insurance industry on the ability of people with unexplained physical symptoms to return to work. If the individual remains highly symptomatic and disabled after vigorous attempts at rehabilitation, it is reasonable to support them.

\section{Preparing medico-legal reports for personal injuries at work}

A common request for those specialising in the management of unexplained physical symptoms is to prepare a medico-legal report for injury that is alleged to have led to chronic unexplained physical symptoms (Box 4). While psychiatrists probably only have a peripheral role in many cases, they may be called upon to assist in difficult decisions. The difficulty psychiatrists face is the need to couch what are often complex, multi-faceted cases within a legal framework which operates in simple 'black and white' terms (Main \& Spanswick, 1995; Wessely, 1995). The key question surrounds causation: did the injury in the workplace lead to the current problems? The key to answering this is through a thorough examination of documentary evidence from before the accident. The best source of such evidence is usually the general practioner's records, which should always be requested and reviewed before examining the patient.
When preparing the report the solicitor will want answers to the following questions.

What is the diagnosis? It is important to be as specific and informative as possible when giving the diagnosis. Use operational criteria when possible and list the symptoms you have elicited to support the diagnosis. It is also worth describing some of the key features of the disorder in the report, emphasising that it is idiopathic, and that there are no laboratory tests to prove it.

Was the accident responsible? In civil cases the burden of proof is the balance of probabilities. This means that there must be reasonable certainty that the accident at work was responsible for the symptoms the person complains of. In most cases

Box 4. Preparing medico-legal reports

Before the interview

Only proceed following written instructions from a solicitor

Remember the report is prepared for the court, not the solicitor; the court will want your impartial professional opinions

Assess all available documentary evidence; always see the general practitioner's notes before seeing the plaintiff

Note any previous history of medically unexplained symptoms and psychiatric disorder

Note current treatments and compliance

\section{At the interview}

Gain a detailed account of the accident, including the plaintiff's reaction to the incident

What are the current symptoms, what disability do they cause, and how does the plaintiff understand them?

What is the mental state and are there any current psychiatric diagnoses?

What is the prognosis and what factors may reduce likelihood of return to work?

When writing the report

Use numbered paragraphs

Describe factual evidence first, including mental state examination

Give opinions separately, with relevant subheadings

Give explicit opinions on diagnosis, causation and prognosis 
this is far from easy to demonstrate. The most helpful approach is to go through the general practice case notes before seeing the plaintiff, and make a note of any episodes of previous psychiatric disorder or other medically unexplained symptoms. In some cases there is a long history of medically unexplained symptoms prior to the accident, and one can be confident that the accident was not responsible for their onset (although plaintiff's solicitors will argue that the accident made matters worse). The psychiatrist must be aware of the implications for the recent Page $v$. Smith judgement (1997). In this case, Page was the victim of a road traffic accident. He had previously suffered from symptoms akin to chronic fatigue syndrome, but had recovered by the time of the accident. Shortly after the accident he experienced many of the old symptoms. The expert witness argued that although the symptoms could not be accounted for by defined organic processes, the relapse was due to 'nervous shock', and Page won. The ruling (and subsequent appeals) was controversial, but rests on the 'eggshell skull' principle. In other words, it is acceptable to argue that pre-existing psychiatric disorder may have predisposed an individual to prolonged illhealth after what would for most people have been a relatively minor injury. On the other hand, insurers may argue that other injuries or life events would have had the same effect sooner or later.

Solicitors often want answers to unanswerable questions. A typical case would be a worker who has a history of depression in the past, may have an unhappy marriage and have had conflict with some authority figure in the workplace, who develops chronic pain following what appears to be a trivial accident. The accident may well have had some part to play, but clearly it is very difficult to know the likelihood of developing symptoms if it had not occurred.

Are there any mitigating circumstances? The most common mitigating circumstance for the defence is delayed and inadequate treatment. It is depressingly common to see someone for a medico-legal report whose accident occurred five years before, but who has never been properly treated. It is important to establish whether the plaintiff has cooperated with any treatment offered.

What is the prognosis? If the plaintiff has received no effective treatment, it is best not to comment on prognosis. If they have received vigorous treatment, it is reasonable to make some attempt to define prognosis, but this often depends on their willingness to return to work.

\section{References}

American Psychiatric Association (1994) Diagnostic and Statistical Manual of Mental Disorders (4th edn)(DSM-IV). Washington, DC: APA.

Aylward, M. \& Locascio, J. J. (1995) Problems in the assessment of psychosomatic conditions in social security benefits and related commercial schemes. Journal of Psychosomatic Reserach, 39, 755-765.

Bigos, S. J., Battie, M. C., Spengler, D. M., et al (1991) A prospective study of work perceptions and psychosocial factors affecting the report of back injury. Spine, 16, 1-6.

Bongers, P. M., de Winter, C. R., Kompier, M. A. J., et al (1993) Psychosocial factors at work and musculoskeletal disease. Scandinavian Journal of Work, Environment and Health, 19, 297-312.

Craig, T. K. J., Boardman, A. P., Mills, K., et al (1993) The South London somatisation study. I: Longitudinal course and the influence of early life experiences. British Journal of Psychiatry, $163,579-588$

Croft, P. R., Papageorgiou, A. C., Ferry, S., et al (1996) Psychologic distress and low back pain: evidence from a prospective study in the general population. Spine, 20, 2731-2737.

Crook, J., Rideout, E. \& Browne, G. (1984) The prevalence of pain complaints in a general population. Pain, 18, 299-14.

Deale, A., Chalder, T., Marks, I., et al (1997) A randomised controlled trial of cognitive behaviour versus relaxation therapy for chronic fatigue syndrome. American Journal of Psychiatry, 154, 408-414.

Dworkin, R. H., Hartstein, G., Rosner, H. L., et al (1992) A high-risk method for studying psychosocial antecedents of chronic pain: the prospective investigation of Herpes Zoster. Journal of Abnormal Psychology, 101, 200-205.

Frank, A. (1993) Low back pain. British Medical Journal, 306, 901-909.

Goldberg, D. P. \& Bridges, K. (1988) Somatic presentation of psychiatric illness in primary care setting. Journal of Psychosomatic Research, 32, 137-144.

Guthrie, E., Creed, F., Dawson, D., et al (1993) A randomised controlled trial of psychotherapy in patients with refractory irritable bowel syndrome. British Journal of Psychiatry, 163, 315-321.

Gwee, K.A., Graham, J. C., McKendrick, M. W., et al (1996) Psychometric scores and persistence of irritable bowel after infectious diarrhoea. Lancet, 347, 150-153.

Hannay, D. R. (1978) Symptom prevalence in the community. Journal of the Royal College of General Practitioners, 28, 492-499.

Hathaway, S. R. \& McKinley, J. C. (1967) Minnesota multiphasic personality inventory: manual for administration and scoring. New York: Psychological Corporation.

Hotopf, M. H., Noah, N. \& Wessely, S. (1996) Chronic fatigue and psychiatric morbidity following viral meningitis: a controlled study. Journal of Neurology, Neurosurgery and Psychiatry, 60, 504-509.

—\& Wessely, S. (1997) Stress in the workplace: unfinished business. Journal of Psychosomatic Research, 43, $1-6$.

Joyce, J., Hotopf, M. \& Wessely, S. (1997) The prognosis of chronic fatigue and chronic fatigue syndrome: a systematic review. Quarterly Journal of Medicine, 90, 223-233.

Kouyanou, K., Pither, C. \& Wessely, S. (1997) Iatrogenic factors in chronic pain: a prospective study. Psychosomatic Medicine, 59, 597-604.

Kroenke, K. \& Mangelsdorff, A. D. (1989) Common symptoms in ambulatory care: incidence, evaluation, therapy, and outcome. American Journal of Medicine, 86, 262-266.

-, Spitzer, R. L., Williams, J. B. W., et al (1994) Physical symptoms in primary care. Archives of Family Medicine, 3, 774-779.

Lipowski, Z. J. (1988) Somatization: the concept and its clinical applications. American Journal of Psychiatry, 145, 1358-1368.

MacFarlane, G. J., Thomas, E., Papageorgiou, A. C., et al (1997) Employment and physical work activities as predictors of future low back pain. Spine, 22, 1143-1149.

Main, C. J. \& Spanswick, C. C. (1995) "Functional overlay", and illness behaviour in chronic pain: distress or malingering? Conceptual difficulties in medico-legal assessment of personal injury claims. Journal of Psychosomatic Research, 39, 737-753. 
Mayou, R., Bass, C. \& Sharpe, M. (1995) Treatment of Functional Somatic Symptoms. Oxford: Oxford University Press.

Mountstephen, A. \& Sharpe, M. (1997) Chronic fatigue syndrome and occupational health. Occupational Medicine, 47, 217-227.

Powell, R., Dolan, R. \& Wessely, S. (1990) Attributions and selfesteem in depression and chronic fatigue syndromes. Journal of Psychosomatic Research, 34, 665-673.

Sharpe, M., Hawton, K., Simkin, S., et al (1996) Cognitive therapy for chronic fatigue syndrome: a randomised controlled trial. British Medical Journal, 312, 22-26.

Svensson, H. O. \& Andersson, G. B. J. (1989) The relationship of low-back pain, work history, work environment, and stress: a retrospective cross-sectional study of 38-64-year-old women. Spine, 14, 517-522.

Wessely, S. (1995) Liability for psychiatric illness. Journal of Psychosomatic Reserach, 39, 659-669.

—\& Powell, R. (1989) Fatigue syndromes: a comparison of chronic "postviral" fatigue with neuromuscular and affective disorders. Journal of Neurology, Neurosurgery and Psychiatry, 52, 940-948.

World Health Organization (1992) The Tenth Revision of the International Classification of Diseases and Related Health Problems. (ICD-10). Geneva: WHO.

\section{Multiple choice questions}

1. Sufferers from unexplained physical symptoms:

a have increased likelihood of psychiatric disorder if they report multiple symptoms

b suffer from a diagnosable psychiatric disorder in the majority of cases

c are more likely to report having had sickly parents during childhood

d always attribute symptoms to organic disease

e may attribute symptoms to organic disease as a defence against guilt.

2. Regarding workplace factors and unexplained physical symptoms:

a accidents in the workplace are cited as the cause of symptoms by over a quarter of sufferers

b the physical mechanisms of sick building syndrome are well understood

c standing and lifting are not associated with back pain d low job satisfaction is associated with sickness absence

e therapy is ineffective following medical retirement.

3. In dealing with patients with medically unexplained symptoms:

a psychiatrists are unlikely to be of any use unless there is a clear psychiatric disorder, such as depression or anxiety

b patients who attribute their symptoms to physical causes should be contradicted and told their problems are psychological

c psychiatrists should avoid asking questions about physical symptoms as this will only reinforce the patient's illness behaviour

d psychiatrists should avoid asking about depression as this will only upset the patient

e it is important to assess the patient's understanding of their illness.

4. Regarding unexplained symptoms:

a most patients are malingering

b only a small minority of patients are dissatisfied with the care they receive

c it is essential to rule out all possible physical causes, no matter how unlikely

$d$ it is useful to repeat investigations which were previously normal 'just to be sure'

e minor pathological change plays no role in the symptoms.

\begin{tabular}{|cccccccc|c|}
\hline \multicolumn{2}{|c|}{ MCQ answers } & & & & & \\
1 & 2 & & & 3 & & & 4 & \\
a & T & a & F & a & F & a & F \\
b & F & b & F & b & F & b & F \\
c & T & c & F & c & F & c & F \\
d & F & d & T & d & F & d & F \\
e & T & e & F & e & T & e & F \\
\hline
\end{tabular}

